\title{
The $\mathrm{AT}_{1}$ receptor antagonist, L-158,809, prevents or ameliorates fractionated whole-brain irradiation-induced cognitive impairment
}

\author{
Mike E. Robbins, Ph.D ${ }^{1,6}, \dagger$, Valerie Payne, B.S ${ }^{1,6}$, Ellen Tommasi, B.S ${ }^{2}$, Debra I Diz, \\ Ph.D2,6, Fang-Chi Hsu, Ph.D ${ }^{3}$, William R. Brown, Ph.D ${ }^{4,6}$, Kenneth T. Wheeler, Ph. ${ }^{4,6}$, John \\ Olson, M.S $\mathbf{S}^{5}$, and Weiling Zhao, Ph. $\mathbf{D}^{1,6}$ \\ 1 Department of Radiation Oncology, Wake Forest University School of Medicine, Winston-Salem, North \\ Carolina 27157
}

2 Hypertension and Vascular Research Center, Wake Forest University School of Medicine, Winston-Salem, North Carolina 27157

3 Department of Biostatistical Sciences, Wake Forest University School of Medicine, Winston-Salem, North Carolina 27157

4 Department of Radiology, Wake Forest University School of Medicine, Winston-Salem, North Carolina 27157

5 Center for Biomolecular Imaging, Wake Forest University School of Medicine, Winston-Salem, North Carolina 27157

6 Brain Tumor Center of Excellence, Wake Forest University School of Medicine, Winston-Salem, North Carolina 27157

\section{Abstract}

Purpose - We hypothesized that administration of the angiotensin type $1\left(\mathrm{AT}_{1}\right)$ receptor antagonist, L-158,809, to young adult male rats would prevent or ameliorate fractionated whole-brain irradiation (WBI)-induced cognitive impairment.

Methods and Materials-Groups of 80 young adult male Fischer $344 \times$ Brown Norway $(\mathrm{F} 344 \times \mathrm{BN})$ rats, $12-14$ weeks old, received either: i] fractionated WBI; 40 Gy of $\gamma$ rays in 4 weeks, 2 fractions/week, ii] sham-irradiation; iii] WBI plus L-158,809 (20 mg/L drinking water) starting 3 days prior, during and for 14,28 , or 54 weeks post-irradiation; and iv] sham-irradiation plus L-158,809 for 14,28 , or 54 weeks post-irradiation. An additional group of rats $(n=20)$ received L-158,809 prior to, during, and for 5 weeks post-irradiation, after which they received normal drinking water up to 28 weeks post-irradiation

$†$ Corresponding author: Mike E. Robbins, PhD, Room 412C NRC, Department of Radiation Oncology, Wake Forest University School of Medicine, Medical Center Blvd, Winston-Salem, NC 27157, Tel. (336) 713-7635, Fax. (336) 713-7639, E-mail: mrobbins@wfubmc.edu.

Conflict of Interest Notification

Actual or potential conflicts of interest do not exist.

Publisher's Disclaimer: This is a PDF file of an unedited manuscript that has been accepted for publication. As a service to our customers we are providing this early version of the manuscript. The manuscript will undergo copyediting, typesetting, and review of the resulting proof before it is published in its final citable form. Please note that during the production process errors may be discovered which could affect the content, and all legal disclaimers that apply to the journal pertain. 
Results-Administration of L-158,809 prior to, during, and for 28 or 54 weeks after fractionated WBI prevented or ameliorated the radiation-induced cognitive impairment observed 26 and 52 weeks post-irradiation. Moreover, giving L-158,809 prior to, during, and for only 5 weeks post-irradiation ameliorated the significant cognitive impairment observed 26 weeks post-irradiation. These radiation-induced cognitive impairments occurred without any changes in brain metabolites or gross histologic changes assessed at 28 and 54 weeks post-irradiation, respectively.

Conclusions-Administering L-158,809 prior to, during, and after fractionated WBI can prevent or ameliorate the chronic, progressive, cognitive impairment observed in rats at 26 and 52 weeks post-irradiation. These findings offer the promise of improving the quality of life for brain tumor patients.

\section{Keywords}

rat brain; fractionated whole-brain irradiation; cognitive impairment; renin-angiotensin system; $\mathrm{L}-158,809 ; \mathrm{AT}_{1}$ receptor antagonists

\section{INTRODUCTION}

For those patients receiving irradiation for primary or metastatic brain tumors, radiationinduced cognitive impairment represents a major morbidity that can markedly impact their quality of life. Progressive cognitive impairment can occur in up to $50 \%$ of brain tumor patients surviving $\geq 6$ months after receiving fractionated partial or whole-brain irradiation [WBI] (1). This is of concern for patients with primary and metastatic brain tumors; the annual incidence is currently estimated at 170,000 (2). Although short-term interventions have proven efficacious (3), there are currently no long-term treatments or preventive approaches for radiation-induced cognitive impairment.

In the last decade or so, experimental studies have demonstrated clearly that radiation-induced late effects are amenable to treatment $(4,5)$. One of the most effective approaches has been blockade of the renin-angiotensin system [RAS] (6). Angiotensin-converting enzyme inhibitors (ACEI) or angiotensin type 1 receptor antagonists $\left(\mathrm{AT}_{1} \mathrm{RA}\right)$ have proved highly effective in the treatment and the prevention of experimental radiation nephropathy $(7,8)$. The ACEI, captopril, and the $\mathrm{AT}_{1} \mathrm{RA}, \mathrm{L}-158,809$, have proven equally useful in protecting against radiation-induced pneumopathy and lung fibrosis $(9,10)$.

The RAS has classically been viewed as a complex systemic hormonal system. More recently, a number of intraorgan RASs have been identified (11). Recent studies have highlighted the importance of the brain RAS in modulating cardiovascular and fluid-electrolyte homeostasis $(12,13)$. Moreover, the RAS is involved in brain-specific functions, including modulation of the blood-brain barrier, stress, memory, and cognition $(13,14)$. Beneficial effects of RAS blockade on cognition have been observed both experimentally and clinically. ACEI attenuate the age-related decline in spontaneously hypertensive rats (15) and normotensive Wistar rats (16). The $\mathrm{AT}_{1} \mathrm{RA}$, losartan, improves cognitive function in hypertensive patients, independent of any reduction in blood pressure (17). These findings suggest an important role for the brain RAS in normal cognitive function and potentially in the treatment of dysfunctional memory disease states (18).

Based on these observations, we hypothesized that blocking the brain RAS would prevent and/ or ameliorate radiation-induced cognitive impairment. The data presented here indicate that administering the $\mathrm{AT}_{1} \mathrm{RA}, \mathrm{L}-158,809$, prior to, during, and for various times up to 54 weeks after fractionated WBI can prevent or ameliorate radiation-induced cognitive impairment in a well-characterized rat model (19). 


\section{METHODS AND MATERIALS}

\section{Animals and WBI procedures}

Groups of 80 young adult (10-12 week old) male Fischer 344 x Brown Norway (F344xBN) rats were obtained from Harlan Sprague Dawley, Inc., (Indianapolis, IN) and housed in pairs on a 12:12 light:dark schedule with free access to food and water. All animal handling and experiments were performed in strict accordance with the Declaration of Helsinki and the NIH Guide for Care and Use of Laboratory Animals as approved by the WFUSM IACUC.

Following an acclimation period of 2 weeks, rats were randomized to four experimental groups $(n=20)$. These consisted of: i] WBI, ii] sham-irradiation, iii] WBI plus the $\mathrm{AT}_{1} \mathrm{RA}, \mathrm{L}-158,809$ (Merck \& Co., Inc., Rahway, NJ; $20 \mathrm{mg} / \mathrm{L}$ drinking water), and iv] sham-irradiation plus $\mathrm{L}-158,809$. Rats received the $\mathrm{L}-158,809$ three days prior to the start of $\mathrm{WBI}$ and were maintained on the $\mathrm{AT}_{1} \mathrm{RA}$ during and after fractionated $\mathrm{WBI}$ for 14,28 or 54 weeks postirradiation. Fresh drinking water with or without L-158,809 was supplied every other day. An additional group of rats $(n=20)$ received L-158,809 prior to, during, and for 5 weeks postirradiation followed by normal drinking water to 28 weeks post-irradiation.

Fractionated WBI (40 Gy, 8 fractions of $5 \mathrm{~Gy}$, twice/week for 4 weeks) was performed as described previously (19). Briefly, all irradiations were performed in a $267 \mathrm{TBq}(7,214 \mathrm{Ci})$ self-shielded ${ }^{137} \mathrm{Cs}$ irradiator using lead and Cerrobend shielding devices to collimate the beam so that the eyes were shielded, and the whole brain, including the brain stem, was irradiated. The average dose rate to the midline of the brain was $\sim 4 \mathrm{~Gy}$; the eyes and body received about $15 \%$ and $1-3 \%$ of the brain dose, respectively. To ensure that each rat received the same midline brain dose, each lightly anesthetized (Ketamine $(75 \mathrm{mg} / \mathrm{Kg}) /$ xylazine $(7 \mathrm{mg} / \mathrm{Kg})$ rat had the twice weekly dose delivered to alternate sides of the head on alternate days. Sham-irradiated rats were anesthetized twice weekly for 4 weeks.

\section{Tissue processing and blood collection}

Rats were weighed weekly for up to 14, 28 or 54 weeks after the completion of fractionatedor sham-WBI, at which time they were euthanized. The brains were removed rapidly and hemisected at the midline. The right hemisphere was flash frozen in liquid nitrogen; the left hemisphere was immersion fixed in phosphate-buffered $4 \%$ paraformaldehyde for $24 \mathrm{~h}$. Brains were then cryoprotected for $24 \mathrm{~h}$ in 10\%, 20\%, and 30\% sucrose and frozen in embedding medium. In animals euthanized at 14 and 54 weeks post-irradiation, blood was collected for analysis of plasma Ang I, Ang II, and Ang-(1-7) using radioimmunoassay (20,21), and for serum glucose using a Freestyle glucose monitor (22).

\section{Histopathology}

Histopathology studies were performed on 12 rats at 54 weeks post-irradiation; three WBI, three sham-irradiated, three WBI plus L-158,809, and three sham-irradiated plus L-158,809 rats. Following fixation, the brain tissue was dehydrated in ethanol and embedded in paraffin. Six sections, $9 \mu \mathrm{m}$ thick, from each of the 12 rats were examined for gross morphological changes after staining with Gill's H\&E, Luxol Fast Blue, and modified Masson's trichrome.

\section{Object recognition test}

Cognitive function was assessed in each animal two weeks prior to their euthanization at 14, 28 , and 54 weeks after the completion of fractionated WBI using the object recognition test, a robust measure of recognition memory in rodents (23). Complete details of the technique have been previously described (24). The following measurements were taken to calculate the discrimination ratio, a measure of the rat's recognition memory (25): $\mathbf{E}_{\mathbf{1}}$ : The total time spent exploring the identical objects $\boldsymbol{A}_{\boldsymbol{I}}$ and $\mathbf{A}_{\mathbf{2}}$ in the sample phase ( 3 min duration; mean values 
for $\mathbf{E}_{\mathbf{1}}$ in animals tested at 12, 26, and 52 weeks after WBI were $26.3 \pm 8.1,26.8 \pm 8.0$, and $20.9 \pm 6.4$ seconds, respectively. $\mathbf{E}_{\mathbf{2}}$ : The total time spent exploring objects $\boldsymbol{A}_{\mathbf{3}}$ (identical to $\boldsymbol{A}_{\boldsymbol{1}}$ and $\mathbf{A}_{\mathbf{2}}$ ) and the novel object $\boldsymbol{B}_{\mathbf{1}}$ in test phase (3 min, the mean $\mathbf{E}_{\mathbf{2}}$ values were 23.6 \pm 9.1, $20.7 \pm 7.7$, and $18.1 \pm 5.7$, respectively); $\mathbf{D}_{\mathbf{1}}$ : Index of discrimination defined as the difference in time spent exploring objects $\mathbf{A}_{\mathbf{3}}$ and $\mathbf{B}_{\mathbf{1}}$ in the test phase, i.e. $\boldsymbol{B}_{\mathbf{1}}-\boldsymbol{A}_{\mathbf{3}} ; \mathbf{D}_{\mathbf{2}}$ : The discrimination ratio, defined as the difference in time spent exploring objects $\boldsymbol{A}_{\mathbf{3}}$ and $\boldsymbol{B}_{\mathbf{1}}$ expressed as a proportion of the total time spent exploring $\boldsymbol{A}_{\mathbf{3}}$ and $\boldsymbol{B}_{\mathbf{1}}$ in the test phase, i.e., $\mathbf{D}_{\mathbf{1}} / \mathbf{E}_{\mathbf{2}}$.

\section{Magnetic resonance imaging (MRI) and spectroscopy (MRS) procedures}

MRI and MRS experiments were carried out 28 weeks post-irradiation as described previously (24) using a horizontal 7T magnet (Bruker BioSpin, Billerica, MA) interfaced with a digital spectrometer operating at a resonant frequency of $300 \mathrm{MHz}$. $\mathrm{T}_{1}$-and $\mathrm{T}_{2}$-weighted images were acquired using continuously-spaced, multi-slice images ( $1 \mathrm{~mm}$ slice thickness) to cover the entire brain with a field of view of $3.5 \mathrm{~cm}$ by $3.5 \mathrm{~cm}$ and a matrix size of 256 by 256 . All images were reviewed by at least two neuroradiologists to detect morphologic changes or lesions in the rat brains.

These high resolution MRI images were also used as localizers for positioning of the MRS voxel. All proton MR spectra were acquired using a single-voxel, double spin-echo point resolved spectroscopy (PRESS) sequence, with TE $=16 \mathrm{~ms}$ and TR $=1800 \mathrm{~ms}$, and a total number of acquisitions of 256. Two separate sets of MR spectra, with and without water suppressions, were acquired from each animal and analyzed as described previously (24).

\section{Statistical analysis}

Data are presented as mean \pm standard error of the mean (SEM). To minimize heterogeneity of variance, we examined the distribution of outcome measures and the need for any transformations to best approximate the normality assumption. One-way analysis of variance (ANOVA) was used to compare the treatment or radiation effect. Bonferroni, Tukey-Kramer, and Student-Newman-Keuls tests were used for the pair wise comparisons; a general linear model was used to determine interactions between the drug and radiation treatments. All analyses were performed using Graphpad Prism (La Jolla, CA) and SAS (Cary, NC) software. For the MRS analysis, the concentration of each metabolite was determined as described previously (24).

\section{RESULTS}

We have previously demonstrated that fractionated WBI in young adult male rats leads to cognitive impairment one year post-irradiation (26). To more accurately define the temporal features of this WBI-induced cognitive impairment, the relative cognitive function was determined at 12, 26 and 52 weeks post-irradiation (Fig. 1A). The mean relative cognitive function was $73.0 \pm 5.5 \%$ at 12 weeks post-irradiation and continued to decrease to $45.0 \pm$ $3.8 \%$ and $14.0 \pm 3.9 \%$ at 26 and 52 weeks, respectively. Thus, irradiating the young adult male rat brain with $40 \mathrm{~Gy}$ of fractionated WBI leads to a chronic, progressive cognitive impairment.

Histological examination of the rat brains obtained 54 weeks after completion of the fractionated WBI (Fig. 1B) revealed no gross morphological changes such as demyelination $(a, b)$, hemorrhage $(c, d)$, or neuronal damage $(e, f)$ ). Thus, these data indicate that fractionated WBI leads to a chronic and progressive cognitive impairment in the absence of any gross histological changes.

L-158,809 did not produce a significant change in the cognitive function of the sham-irradiated controls (Fig. 2). In contrast, administering L-158,809 prior to, during, and after fractionated 
WBI prevented or ameliorated the radiation-induced cognitive impairment at all of the time points (Fig. 2). Moreover, administration of L-158,809 prior to, during, and for only 5 weeks after WBI also reduced the radiation-induced cognitive impairment observed at 26 weeks postirradiation (Fig. 3). Thus, administering L-158,809 prior to, during, and for as little as 5 weeks post-irradiation can ameliorate WBI-induced cognitive impairment.

As previously described at 12 months post-irradiation (24), high-resolution magnetic resonance imaging (MRI) of the rat brain at 6 months post-irradiation revealed no apparent $T_{1}$ or $T_{2}$ lesions (data not shown). We previously showed that the ratio of $\mathrm{N}$-acetyl aspartate (NAA), glutamine + glutamate $(\mathrm{Glx})$ and myoinositol $(\mathrm{mI})$ normalized to total creatine $(\mathrm{tCr})$ were significantly altered in young adult male F344 rats at 12 months after 40 Gy of fractionated WBI (24). However, no alteration in these brain metabolite ratios was measured in the cognitively impaired rats at 26 weeks post-irradiation in this study (Table 1). Thus, postirradiation changes in brain metabolites may be associated with WBI-induced cognitive impairments, but they appear to be neither causative nor predictive of these cognitive impairments.

The mean body weight (BW) of rats treated with fractionated WBI was significantly lower than that of the sham-irradiated controls at 14 and 28 weeks post-irradiation (Fig. 4A). This decreased BW was also noted in the rats receiving L-158,809, with or without WBI. Although the sham-irradiated + L-158,809 rats still had a small, but statistically significant, decrease in BW at 54 weeks, the BWs of the WBI with or without L-158,809 were essentially identical to the sham-irradiated controls (Fig. 4A). Serum glucose levels were significantly decreased in WBI rats treated with or without L-158,809 at 14 weeks post-irradiation (Fig. 4B). However, the serum glucose levels were similar in all of the groups by 54 weeks post-irradiation (Fig. 4B). Taken together, these data indicate that fractionated WBI with or without L-158,809 has little adverse effect on the overall health status of these rats up to 1 year post-irradiation.

The plasma angiotensin (Ang) peptide levels of Ang I, Ang II, and Ang-(1-7) were increased in the rats receiving L-158,809 for 14 or 54 weeks regardless of whether they were irradiated or not (Fig. 5). In contrast, no significant differences in the levels of these Ang peptides were observed between sham-irradiated and WBI rats that did not receive L-158,809 (Fig. 5). Thus, chronic administration of L-158,809 was associated with a persistent increase in plasma Ang peptides.

\section{DISCUSSION}

The current data demonstrate that irradiating the brains of young adult male F344xBN rats with a fractionated dose of $40 \mathrm{~Gy}$ of $\gamma$ rays leads to a chronic, progressive, reduction in cognitive function that is statistically significant at 26 and 52 weeks post-irradiation. Administering the $\mathrm{AT}_{1} \mathrm{RA}, \mathrm{L}-158,809$, prior to, during, and after completion of fractionated WBI prevented this radiation-induced cognitive impairment. Moreover, administering the L-158,809 for only 5 weeks post-irradiation ameliorated the radiation-induced cognitive impairment. Chronic administration of L-158,809 was associated with significant increases in systemic levels of Ang I, Ang II, and Ang-(1-7), indicating that the $\mathrm{AT}_{1} \mathrm{R}$ blockade was effective. Thus, as hypothesized, long-term $\mathrm{AT}_{1} \mathrm{R}$ blockade with $\mathrm{L}-158,809$ appears to prevent radiation-induced cognitive impairment.

The observation that fractionated WBI of the young adult male rat leads to a significant reduction in cognitive function 26 and 52 weeks post-irradiation extends previous studies $(26,27)$. Using a partially baited radial-arm maze (28), Brown et al (27) noted a significant reduction in cognitive function 6 and 9 months after 40 Gy of fractionated WBI. The current study used the non-hippocampal-dependent novel object recognition task to assess recognition 
memory; this was significantly impaired 26 and 52 weeks post-irradiation. Thus, fractionated WBI leads to significant and progressive reductions in both hippocampal- and nonhippocampal-dependent cognitive function, suggesting that multiple regions of the brain play a role in radiation-induced cognitive impairment.

This chronic and progressive decline in cognitive function was prevented by administration of the $\mathrm{AT}_{1} \mathrm{RA}, \mathrm{L}-158,809$, prior to, during, and after the completion of fractionated WBI. These data appear to be the first to demonstrate that an $\mathrm{AT}_{1} \mathrm{RA}$ can prevent radiation-induced cognitive impairment. Moreover, this was also observed in rats that received L-158,809 prior to, during, and for only 5 weeks after WBI. These findings are similar to those using the antiinflammatory peroxisomal proliferator-activated receptor (PPAR) $\gamma$ agonist, Pioglitazone (Pio) (26). Administration of Pio before, during, and for 4 weeks was as effective as giving the drug for 54 weeks at preventing the radiation-induced cognitive impairment measured at 12 months after WBI. In contrast, starting Pio treatment after the completion of WBI and maintaining it for 54 weeks proved relatively ineffective. Thus, at least in the young adult male rat, prevention or amelioration of radiation-induced cognitive impairment appears to require administration of the drug prior to, during and perhaps for only $4-5$ weeks after WBI.

Modulation of the RAS has been shown previously to mitigate the severity of radiation-induced CNS injury $(29,30)$. Chronic administration of the ACEI, ramipril, initiated two weeks after stereotactic irradiation of the rat brain with a single dose of $30 \mathrm{~Gy}$, was associated with a reduction in the severity of functional and histopathologic markers of optic neuropathy assessed 6 months post-irradiation (29). However, delaying the start of ramipril treatment to 4 weeks after irradiation resulted in a failure to reduce the severity of the radiation injury (30). Similarly, administering the $\mathrm{AT}_{1} \mathrm{RA}$, losartan, (an analog of L-158,809 used clinically as an anti-

hypertensive agent) starting two weeks post-irradiation did not mitigate the radiation-induced optic neuropathy (29). Ryu et al suggested that this may be related to limited drug availability in the brain (30). However, this seems unlikely given that the concentration of L-158,809 used in the current study $(2 \mathrm{mg} / \mathrm{kg} /$ day $)$ was only $10 \%$ of that used in the optic neuropathy model. Moreover, the marked increase in systemic levels of Ang I, Ang II and Ang-(1-7) observed in our rats treated with L-158,809 for 14 or 54 weeks is consistent with previous observations (22) of effective $\mathrm{AT}_{1} \mathrm{R}$ blockade, and indicates loss of the RAS feedback mechanism (31). Further studies are required to help explain these apparent differences in effectiveness of ACEI and $\mathrm{AT}_{1} \mathrm{RA}$ treatment in these models of radiation-induced CNS injury.

The reduction in cognitive function seen following fractionated WBI in the rat occurs in the absence of gross histological changes. The lack of gross structural alterations reported here in the brains of rats exhibiting significant cognitive impairment confirms previous observations $(19,24)$. High-resolution MRI of the rat brain 26 weeks post-irradiation also revealed no apparent $\mathrm{T}_{1}$ or $\mathrm{T}_{2}$ lesions (data not shown). These findings confirm and extend previous studies in which MRI revealed no apparent lesions or changes in $\mathrm{T}_{1}$ - or $\mathrm{T}_{2}$-weighted images at 14 and 54 weeks post-irradiation (24). However, at 54 weeks post-irradiation, MRS revealed significant differences in the concentrations of several brain metabolites between irradiated and sham-irradiated controls, including increases in the NAA/tCr, and $\mathrm{Glx} / \mathrm{tCr}$ ratio, as well as a decrease in the $\mathrm{mI} / \mathrm{tCr}$ ratio (24). These findings suggested that MRS may represent a sensitive, noninvasive tool for detecting changes in radiation-induced brain metabolites that might be associated with fractionated WBI-induced cognitive impairment. However, as reported here, analysis of MR spectra obtained 26 weeks after WBI showed no significant differences in the $\mathrm{NAA} / \mathrm{tCr}$, Glx/tCr or $\mathrm{mI} / \mathrm{tCr}$ ratios determined in WBI rats versus agematched sham-irradiated controls (Table 1). Indeed, levels of these brain metabolites were also not significantly different from those observed in WBI or sham-irradiated rats treated with L-158.809. Thus, changes in rat brain metabolites occur after the expression of radiation- 
induced cognitive impairment, and do not appear to cause or predict the onset of radiationinduced cognitive impairment.

The mechanistic basis for L-158,809-mediated prevention or amelioration of radiation-induced cognitive impairment is unclear. A growing body of evidence supports a causative role for oxidative stress and chronic inflammation in radiation-induced late effects (32). We have hypothesized that the efficacy of RAS blockers reflects inhibition of ongoing interactions between radiation and Ang II that contribute to an aberrant wound healing response (6). Thus, inhibition of an intrinsic organ-based RAS can be viewed as affecting anti-inflammatory processes (6). However, defining the pathogenic mechanisms involved in $\mathrm{AT}_{1} \mathrm{RA}$-mediated prevention or amelioration of radiation-induced cognitive impairment will require more indepth analyses, including identifying putative molecular mediators and signaling pathways.

In summary, administering the $\mathrm{AT}_{1} \mathrm{RA}, \mathrm{L}-158,809$, prior to, during, and for 5,26 or 52 weeks after the completion of fractionated WBI prevented or ameliorated the radiation-induced cognitive impairment seen 26 and 52 weeks post-irradiation. $\mathrm{AT}_{1} \mathrm{RAs}$ are: i] routinely prescribed for the treatment of hypertension (33), ii] well-tolerated, and iii] exhibit antitumor effects (34). Thus, they appear to be ideal drugs for future clinical trials because they offer the promise of improving the quality of life of brain tumor patients who receive fractionated WBI.

\section{Acknowledgements}

This work was supported by NIH grant CA122318, CA113321 and HL51952, as well as an award from Elekta, Inc. The authors would like to thank Dr. Ron Smith of Merck for kindly providing the L-158,809.

Financial support: CA122318 (MER), HL51952 (DIZ), CA113321 (WRB), and Elekta (MER)

\section{References}

1. Meyers CA, Brown PD. Role and relevance of neurocognitive assessment in clinical trials of patients with CNS tumors. J Clin Oncol 2006;24:1305-1309. [PubMed: 16525186]

2. Li J, Bentzen SM, Li J, Renschler M, Mehta MP. Relationship between neurocognitive function and quality of life after whole-brain radiotherapy in patients with brain metastasis. Int J Radiat Oncol Biol Phys 2008;71:64-70. [PubMed: 18406884]

3. Shaw EG, Rosdahl R, D'Agostino RB, et al. Phase II study of donepezil in irradiated brain tumor patients: Effect on cognitive function, mood, and quality of life. J Clin Oncol 2006;24:1415-1420. [PubMed: 16549835]

4. Coleman CN, Stone HB, Moulder JE, Pellmar TC. Modulation of radiation injury. Science 304:693694. [PubMed: 15118152]

5. Bentzen SM. Preventing or reducing late side effects of radiation therapy: radiobiology meets molecular pathology. Nat Rev Cancer 2006;6:702-713. [PubMed: 16929324]

6. Robbins ME, Diz DI. Pathogenic role of the renin-angiotensin system in modulating radiation-induced late effects. Int J Radiat Oncol Biol Phys 2006;64:6-12. [PubMed: 16377409]

7. Cohen EP, Fish BL, Moulder JE. The renin-angiotensin system in experimental radiation nephropathy. J Lab Clin Med 2002;139:251-257. [PubMed: 12024113]

8. Moulder JE, Fish BL, Cohen EP. ACE inhibitors and AII receptor antagonists in the treatment and prevention of bone marrow transplant nephropathy. Curr Pharm Des 2003;9:737-749. [PubMed: 12570791]

9. Ward WF, Molteni A, Ts'ao C, Hinz JM. Captopril reduces collagen and mast cell accumulation in irradiated rat lung. Int J Radiat Oncol Biol Phys 1990;19:1405-1409. [PubMed: 2262365]

10. Molteni A, Moulder JE, Cohen EP, et al. Control of radiation-induced pneumopathy and lung fibrosis by angiotensin-converting enzyme inhibitors and an angiotensin II type 1 receptor blocker. Int $\mathrm{J}$ Radiat Biol 2000;76:523-532. [PubMed: 10815633] 
11. Davisson RL. Physiological genomic analysis of the brain renin-angiotensin system. Am J Physiol Regul Integr Comp Physiol 2003;285:R498-R511. [PubMed: 12909574]

12. Culman J, Blume A, Gohlke P, Unger T. The renin-angiotensin system in the brain: possible therapeutic implications for $\mathrm{AT}_{1}$-receptor blockers. J Human Hypertens 2002;16:S64-S70. [PubMed: 12140731]

13. McKinley MJ, Albiston AL, Allen AM, et al. The brain renin-angiotensin system: location and physiological roles. Int J Biochem Cell Biol 2003;35:901-918. [PubMed: 12676175]

14. Gard PR. The role of angiotensin II in cognition and behaviour. Eur J Pharmacol 2002;438:1-14. [PubMed: 11906704]

15. Wyss JM, Kadish I, van Groen T. Age-related decline in spatial learning and memory: attenuation by captopril. Clin Exp Hypertens 2003;25:455-474. [PubMed: 14596369]

16. Basso N, Paglia N, Stella I, et al. Protective effect of the inhibition of the renin-angiotensin system on aging. Regul Pept 2005;128:247-252. [PubMed: 15837534]

17. Tedesco MA, Ratti G, Di Salvo G, Natale F. Does the angiotensin II receptor antagonist losartan improve cognitive function? Drugs Aging 2002;19:723-732. [PubMed: 12390049]

18. Wright JW, Harding J. The brain angiotensin system and extracellular matrix molecules in neuralplasticity, learning, and memory. Prog Neurobiol 2004;72:263-293. [PubMed: 15142685]

19. Brown WR, Thore CR, Moody DM, Robbins ME, Wheeler KT. Vascular damage after fractionated whole-brain irradiation in rats. Radiat Res 2005;164:662-668. [PubMed: 16238444]

20. Allred AJ, Chappell MC, Ferrario CM, Diz DI. Differential actions of renal ischemic injury on the intrarenal angiotensin system. Am J Physiol Renal Physiol 2000;279:F636-F645. [PubMed: 10997913]

21. Iyer SN, Chappell MC, Averill DB, Diz DI, Ferrario CM. Vasodepressor actions of angiotensin-(17) unmasked during combined treatment with linopril and losartan. Hypertension 1998;31:699-705. [PubMed: 9461243]

22. Gilliam-Davis S, Payne VS, Kasper SO, et al. Long-term $\mathrm{AT}_{1}$ receptor blockade improves metabolic function and provides renoprotection in Fischer 344 rats. Am J Physiol Heart Circ Physiol 2007;293:H1327-H1333. [PubMed: 17616746]

23. Clark RE, Martin SJ. Interrogating rodents regarding their object and spatial memory. Curr Opin Neurobiol 2005;15:593-598. [PubMed: 16150589]

24. Atwood T, Payne VS, Zhao W, et al. Quantitative magnetic resonance spectroscopy reveals a potential relationship between radiation-induced changes in rat brain metabolites and cognitive impairment. Radiat Res 2007;168:574-581. [PubMed: 17973545]

25. Ennaceur A, Delacour J. A new one-trial test for neurobiological studies of memory in rats. 1 : Behavioral data. Behav Brain Res 1988;31:47-59. [PubMed: 3228475]

26. Zhao W, Payne V, Tommasi E, et al. Administration of the peroxisomal proliferator-activated receptor (PPAR) $\gamma$ agonist pioglitazone during fractionated brain irradiation prevents radiation-induced cognitive impairment. Int J Radiat Oncol Biol Phys 2007;67:6-9. [PubMed: 17189061]

27. Brown WR, Blair RM, Moody DM, et al. Capillary loss precedes cognitive impairment induced by fractionated whole-brain irradiation. J Neurol Sci 2007;257:67-71. [PubMed: 17316691]

28. Foreman, N.; Ermakova, E. The radial arm maze: twenty years of the radial arm maze. In: Foreman, N.; Gillett, R., editors. A handbook of spatial research paradigms and methodologies. Hove UK: Psychology Press; 1998. p. 87-143.

29. Kim JH, Brown SL, Kolozsvary A, et al. Modification of radiation injury by ramipril, inhibitor of angiotensin-converting enzyme, on optic neuropathy in the rat. Radiat Res 2004;161:137-142. [PubMed: 14731077]

30. Ryu S, Kolozsvary A, Jenrow KA, Brown SL, Kim JH. Mitigation of radiation-induced optic neuropathy in rats by ACE inhibitor ramipril: importance of ramipril dose and treatment time. J Neurooncol 2007;82:119-124. [PubMed: 17004100]

31. Kasper SO, Basso N, Kurnjek ML, et al. Divergent regulation of circulating and intrarenal reninangiotensin systems in response to long-term blockade. Am J Nephrol 2005;25:335-341. [PubMed: 15976496]

32. Robbins MEC, Zhao W. Chronic oxidative stress and radiation-induced late normal tissue injury: a review. Int J Radiat Biol 2004;80:251-259. [PubMed: 15204702] 
33. Ribeiro AB. Angiotensin II antagonists-therapeutic benefits spanning the cardiovascular disease continuum from hypertension to heart failure and diabetic nephropathy. Curr Med Res Opin 2006;22:1-16. [PubMed: 16393425]

34. Molteni A, Ward WF, Ts'ao C, et al. Cytostatic properties of some angiotensin I converting enzyme inhibitors and of angiotensin II type I receptor antagonists. Curr Pharm Des 2003;9:751-761. [PubMed: 12570792] 
A.

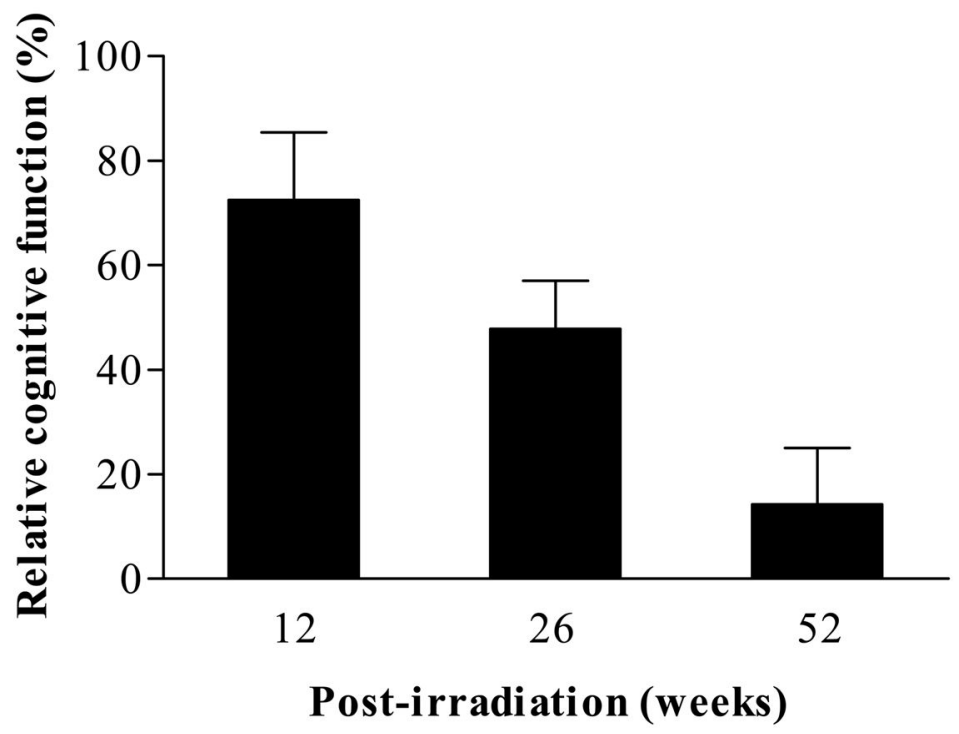

B.

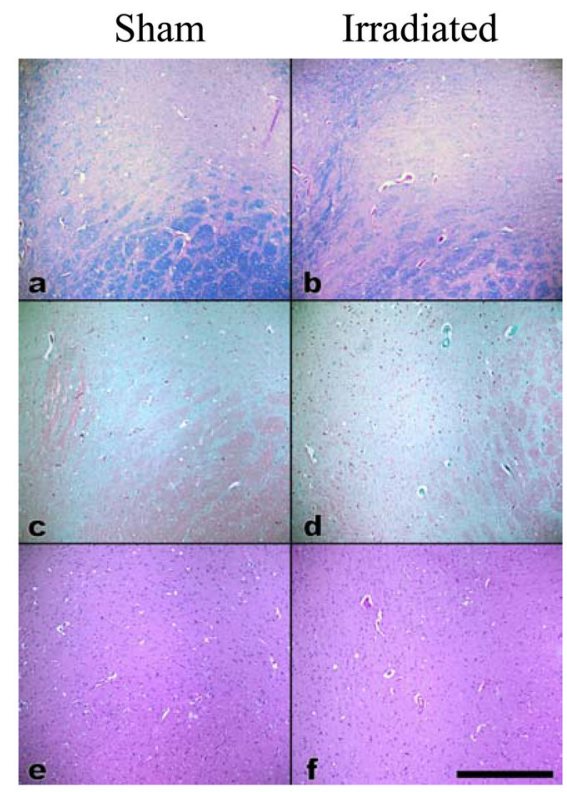

Fig. 1.

Fractionated whole-brain irradiation (WBI; 40 Gy given as eight fractions of 5 Gy, twice/week) of young adult male $\mathrm{F} 344 \mathrm{xBN}$ rats leads to a chronic, progressive decrease in cognitive function in the absence of gross morphological changes. A: Cognitive function was assessed at 12, 26 and 52 weeks post-irradiation using the object recognition task. The mean relative cognitive function \pm 1 SEM $(n=13-20)$ was calculated from the discrimination ratio for each rat expressed as a percentage of the averaged discrimination ratio of the appropriate age-matched sham-irradiated controls. B: Histological sections $(9 \mu \mathrm{m})$ showing no gross morphological changes in irradiated (b, d, f) compared to sham (a, c, e) rat brains at 54 weeks post-irradiation. The stains are Luxol Fast Blue for demyelination ( $a, b)$, modified Masson's trichrome for hemorrhage (c, d) and Gill's H\&E for neuronal damage (e, f). The bar in $\mathrm{f}$ is $500 \underline{\mu \mathrm{m}}$. 
A.

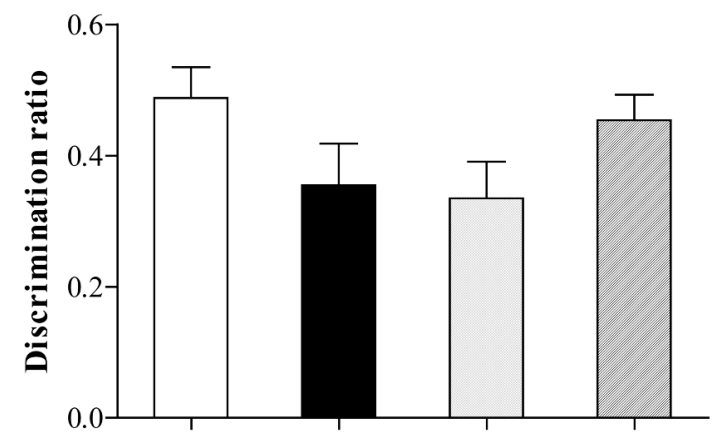

B.

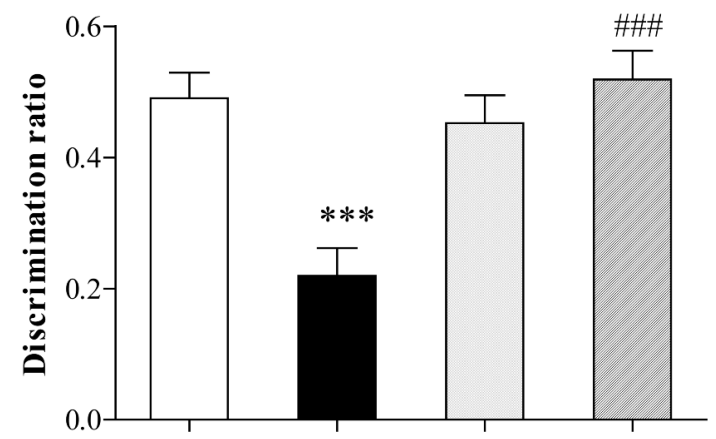

C.

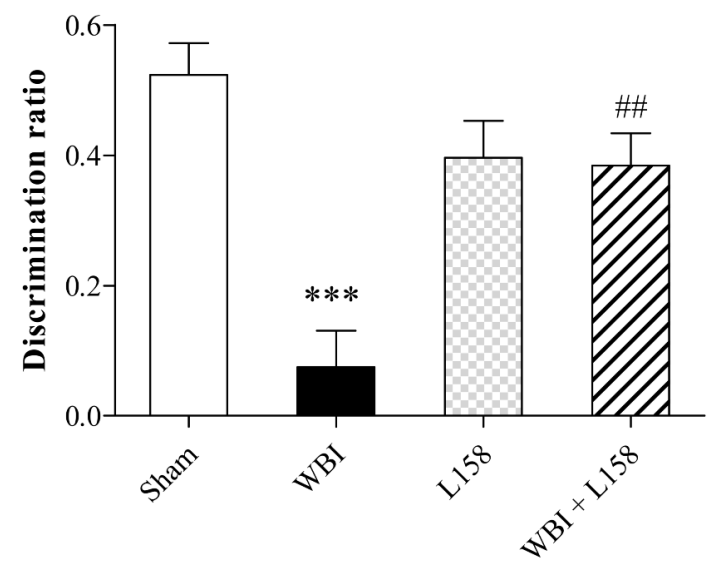

Fig. 2.

Administration of the $\mathrm{AT}_{1} \mathrm{RA}, \mathrm{L}-158,809$, to young adult male $\mathrm{F} 344 \mathrm{xBN}$ rats prior to, during and after fractionated WBI prevents or ameliorates radiation-induced cognitive impairment. Rats received either: i] sham-irradiation, ii] 40 Gy of fractionated WBI, iii] sham-irradiation + L-158,809 (20 mg/L drinking water), or iv] 40 Gy of fractionated WBI + L-158,809. Rats were placed on L-158,809 three days prior to the start of WBI and maintained on the drug for 14 (A), 28 (B), or 54 (C) weeks post-irradiation and cognitive function was assessed at 12, 26, and 52 weeks, respectively. Data represent the mean \pm SEM; $\mathrm{n}=13-20 ; * * * p<0.001$, WBI vs sham-irradiated; \#\# $p<0.01$, \#\#\# $p<0.001$, WBI vs WBI + L-158,809. 


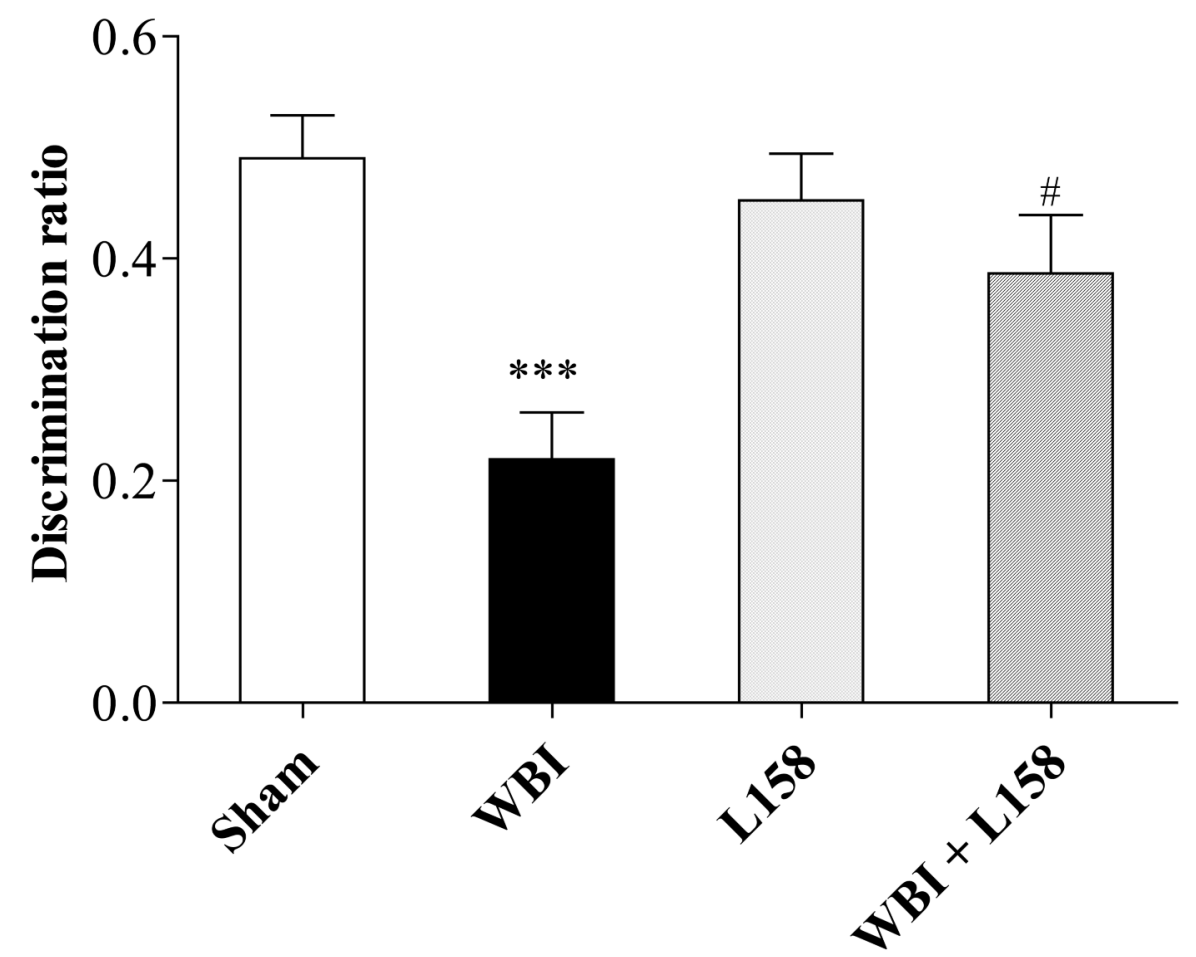

Fig. 3.

Administering the $\mathrm{AT}_{1} \mathrm{RA}, \mathrm{L}-158,809$, to young adult male $\mathrm{F} 344 \mathrm{xBN}$ rats prior to, during and for only 5 weeks after fractionated WBI ameliorates radiation-induced cognitive impairment at 26 weeks post-irradiation. Rats received either: i] sham-irradiation, ii] $40 \mathrm{~Gy}$ of fractionated WBI, iii] sham-irradiation + L-158,809 (20 mg/L drinking water), or iv] 40 Gy of fractionated WBI + L-158,809. Rats were placed on L-158,809 three days prior to the start of WBI and maintained on the drug for 5 weeks post-irradiation. Data represent the mean \pm SEM; $\mathrm{n}=18$ 20; *** $p<0.001$, WBI vs sham-irradiated; \# $p<0.05$, WBI vs WBI + L-158,809. 
A.

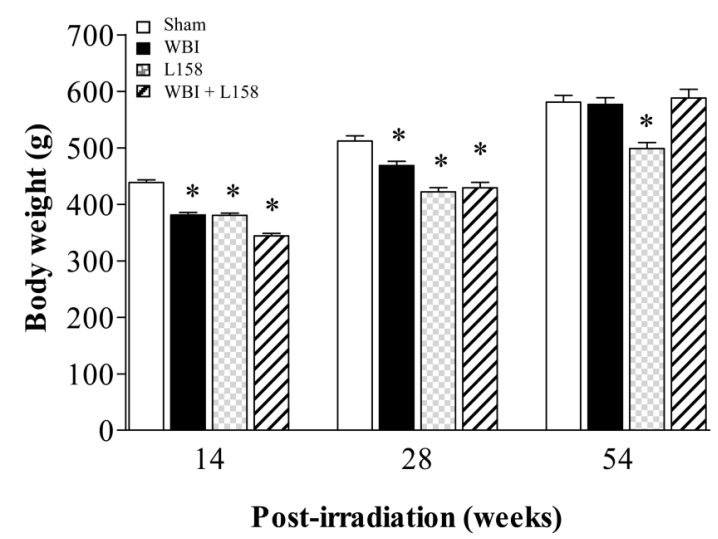

B.

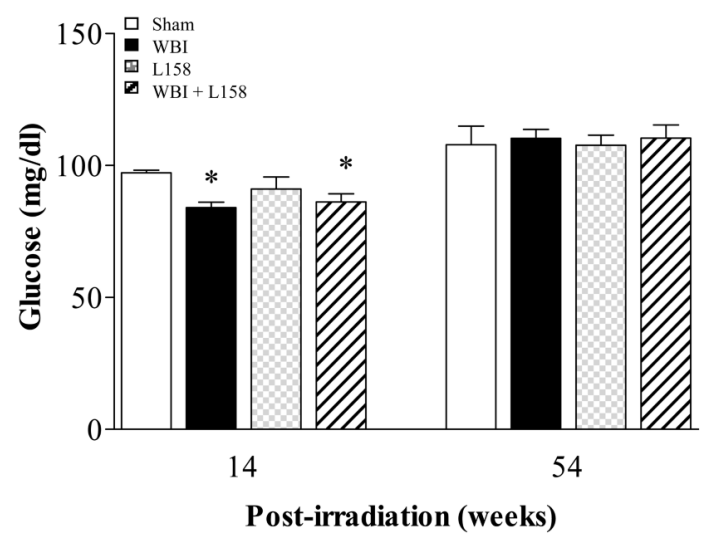

Fig. 4.

Body weight (A) and serum glucose levels (B) determined at 14, 28 and 54 weeks after fractionated WBI. Young adult male F344xBN rats received either: i] sham-irradiation, ii] 40 Gy of fractionated WBI, iii] sham-irradiation + L-158,809 (20 mg/L drinking water), or iv] 40 Gy of fractionated WBI + L-158,809. Rats were placed on the L-158,809 three days prior to the start of WBI and maintained on the drug for the duration of the experiment. Data represent A. Mean \pm SEM; $n=18-20 ; B$. Mean \pm SEM, $n=6-8 . *$ Represents values significantly different from those observed in the appropriate age-matched sham-irradiated controls; $p<$ 0.05 . 
A.

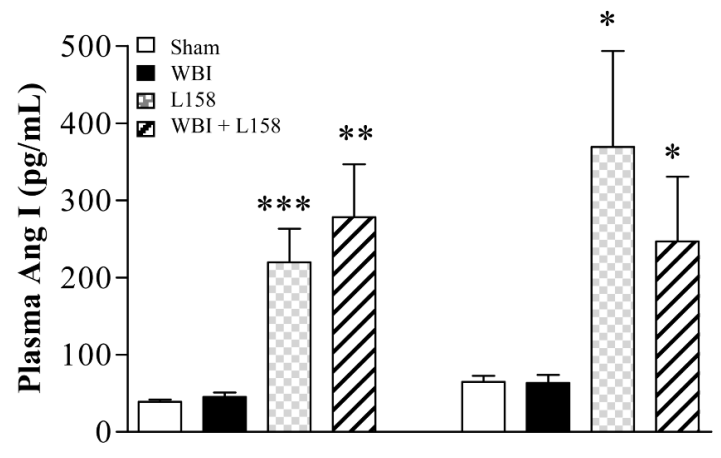

B.
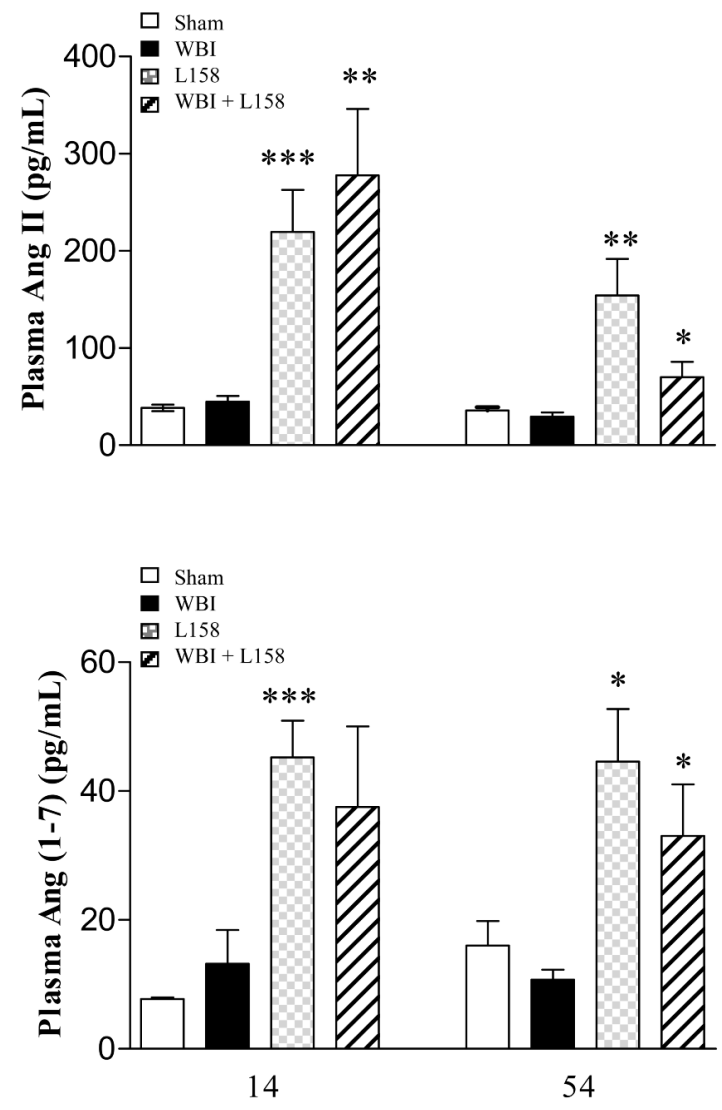

Post-irradiation (weeks)

Fig. 5.

Chronic administration of L-158,809 is associated with a marked increase in plasma angiotensin (Ang) peptides. Young adult male F344xBN rats received either: i] shamirradiation, ii] 40 Gy of fractionated WBI, iii] sham-irradiation + L-158,809 $(20 \mathrm{mg} / \mathrm{L}$ drinking water), or iv] 40 Gy of fractionated WBI + L-158,809. Rats were placed on L-158,809 three days prior to the start of WBI and maintained on the drug for the duration of the experiment. Plasma Ang peptide levels were determined when the rats were euthanized at 14 or 54 weeks post-irradiation. A; Ang I; B: Ang II, C: Ang-(1-7). Values of Ang II in the sham-irradiation controls are similar to those reported previously $(22,31)$; values for Ang-(1-7) have not been reported previously in $\mathrm{F} 344 \mathrm{xBN}$ rats. Data represent mean $\pm \mathrm{SEM} ; \mathrm{n}=6-8 ; * p<0.05$, ** $p$ 
$<0.01, * * * p<0.001, \mathrm{~L}-158,809 \mathrm{w} / \mathrm{wo} \mathrm{WBI}$ vs age-matched sham-irradiated or WBI, respectively. 
Table 1

Brain metabolite concentration ratios 26 weeks after fractionated whole-brain irradiation

\begin{tabular}{ccc}
\hline Metabolic ratio & Sham-irradiation & Whole-brain irradiation \\
\hline NAA/tCr & $1.17 \pm 0.07$ & $1.14 \pm 0.09$ \\
Glx/tCr & $1.84 \pm 0.22$ & $2.02 \pm 0.19$ \\
$\mathrm{mI} / \mathrm{tCr}$ & $0.93 \pm 0.08$ & $1.04 \pm 0.08$ \\
\hline
\end{tabular}

$\mathrm{NAA}=\mathrm{N}$-acetyl aspartate $; \mathrm{Glx}=$ Glutamine and glutamine $\mathrm{mI}=$ myoinositol $; \mathrm{tCr}=$ creatine + phosphocreatine. Data are mean $\pm \mathrm{SD} ; \mathrm{n}=5$ 